\title{
Effect of ramipril on $\alpha$-adrenoceptor-mediated oscillatory contractions in tail artery of hypertensive rats
}

\author{
Stephanie W. Watts *, Oren Traub, Fred S. Lamb, J. Hurley Myers and R. Clinton Webb \\ Department of Physiology, The University of Michigan, Ann Arbor, MI 48109, USA
}

Received 18 March 1993, revised MS received 15 July 1993, accepted 20 July 1993

\begin{abstract}
Recent studies indicate that norepinephrine-induced contractile oscillations in the tail artery from stroke-prone spontaneously hypertensive rats (SHRSP) may be a vascular phenomenon independent of blood pressure level. The objectives of this study were: (1) to characterize pharmacologically the $\alpha$-adrenoceptor mediating norepinephrine-induced oscillations in tail artery; and (2) to investigate the relationship between blood pressure level, altered by treatments with hydralazine/ hydrochlorothiazide or the angiotensin converting enzyme inhibitor ramipril, and the observation of norepinephrine-induced oscillations in tail artery. The $\alpha_{2}$-adrenoceptor agonists clonidine and guanabenz potently stimulated oscillatory contractions in the tail artery while the $\alpha_{1}$-adrenoceptor agonists phenylephrine and methoxamine were considerably less potent. Yohimbine, an $\alpha_{2}$-adrenoceptor antagonist, but not the $\alpha_{1}$-adrenoceptor antagonist prazosin demonstrated high affinity for the receptor mediating norepinephrine-induced oscillatory contractions. These results support the hypothesis that norepinephrine-induced oscillatory contractions in the tail artery from SHRSP occur primarily through stimulation of $\alpha_{2}$-adrenoceptors. Ramipril lowered blood pressure in SHRSP after 4 weeks of treatment during 6-10 weeks of life but did not alter the ability of the $\alpha_{2}$-adrenoceptor agonist clonidine $\left(10^{-5} \mathrm{M}\right)$ to induce contractile oscillations in tail arteries from SHRSP, indicating these oscillations are not a secondary effect of high blood pressure. These studies suggest that norepinephrine-induced oscillations in tail artery from SHRSP may be a vascular trait separate and distinct from blood pressure level and angiotensin II expression early in life.
\end{abstract}

Ramipril; Adrenoceptors; Genetic hypertension; Oscillation

\section{Introduction}

Hypertension, or elevated blood pressure, is in part effected by changes in vascular smooth muscle. The activity of vascular tissue ultimately affects total peripheral resistance, which in turn determines blood pressure. Several vascular changes have been demonstrated in hypertensive individuals. For example, in many forms of hypertension, blood vessels demonstrate hyperreactivity to contractile agonists including norepinephrine, serotonin and angiotensin II when compared to vessels from normotensive subjects (Bohr and Webb, 1988). It has been difficult, however, to separate changes in vascular activity which occur because of hypertension from changes that are present prior to elevation in blood pressure. Indeed, elevated blood pressure can result in such physical changes as hypertrophy of vascular smooth muscle (Stills, 1979; Gab-

\footnotetext{
* Corresponding author. Tel. (313) 763-5606, fax (313) 936-8813.
}

bianai et al., 1979; Owens and Schwartz, 1982; Grunwald et al., 1982) and basement membrane thickening (Guyton et al., 1990).

Recently, a vascular trait has been identified which is unique to stroke-prone spontaneously hypertensive rats (SHRSP) and may be disparate from the development of hypertension (Bruner et al., 1986; Bruner and Webb, 1987; Myers et al., 1985; Lamb et al., 1985). The addition of norepinephrine to the isolated tail artery of SHRSP results in a steady contraction with superimposed contractile oscillations. This isometric response contrasts with the maintained contraction norepinephrine induces in tail arteries from normotensive Wistar-Kyoto (WKY) rats. In the first part of this study, we present the in vitro pharmacological characterization of the adrenoceptor mediating norepinephrine-induced oscillatory contractions in tail artery from SHRSP.

More importantly, recent evidence also suggests that the oscillatory activity induced in the tail artery of SHRSP may be genetically associated with blood pres- 
sure (Bruner et al., 1986). It is unclear whether the appearance of norepinephrine-induced oscillations in tail arteries from SHRSP signifies an underlying defect in the tissue of these hypertensive rats or whether it is a vascular trait secondary to increased blood pressure. Thus, we have treated a group of SHRSP and normotensive WKY rats with the angiotensin converting enzyme inhibitor ramipril to lower blood pressure; the tail arteries from these animals were then removed and tested for norepinephrine-induced oscillations. Our hypothesis was that if norepinephrine-induced oscillations were secondary to blood pressure level, treatment with a compound which lowered blood pressure should abolish norepinephrine-induced oscillations in tail arteries from SHRSP. This group of experiments also enabled us to test another hypothesis, one recently proposed by Harrap et al. (1990). Harrap et al. (1990) proposed that in genetically hypertensive rats, it is during a critical period of life - 2-10 weeks - that a gene may be 'switched' by angiotensin II, ultimately leading to production of hypertension. Angiotensin converting enzyme inhibitors such as perindopril and ramipril (Todd and Benfield, 1990) inhibit the production of angiotensin II and may therefore permanently lower blood pressure by altering early-life gene 'switching'. If norepinephrine-induced tail artery oscillations in SHRSP are dependent upon angiotensin II production and independent of blood pressure level, then treatment of SHRSP with ramipril but not hydrochlorothiazide / hydralazine - a treatment used to lower blood pressure level independent of angiotensin II production - should diminish the ability of norepinephrine to induce tail artery oscillations. Thus, the present studies investigate the relationship between vascular changes and blood pressure level in genetically hypertensive animals.

\section{Materials and methods}

\subsection{Animals}

Male Wistar Kyoto (WKY) and stroke-prone spontaneously hypertensive (SHRSP) rats were obtained from a colony established in the Department of Anatomy and Cell Biology at the University of Michigan (Ann Arbor, MI, USA). Rats were housed in a light-cycled, temperature-controlled room with ad libitum access to food and water.

Six different treatment groups were used: (1) untreated WKY; (2) untreated SHRSP; (3) hydrochlorothiazide/hydralazine-treated WKY; (4) hydrochlorothiazide/ hydralazine-treated SHRSP; (5) ramipriltreated WKY; and (6) ramipril-treated SHRSP. Hydralazine, a vasodilator, and the diuretic hydrochlorothiazide were used in combination to lower blood pres- sure in a manner independent of angiotensin II production. Rats in the hydrochlorothiazide/hydralazinetreated group received hydralazine $(200 \mathrm{mg} / \mathrm{l})$ and hydrochlorothiazide $(200 \mathrm{mg} / \mathrm{l})$ in their drinking water (deionized water) from 6 to 10 weeks of age. Based on an intake of $20 \mathrm{ml}$ of water per day, hydrochlorothiazide/hydralazine-treated rats received $4 \mathrm{mg}$ /day of each drug. All other rats received tap water. After treatment from 6 to 10 weeks of age, the hydrochlorothiazide/hydralazine-treated rats were switched to tap water for the remainder of the experiment. Rats in the ramipril-treated group received ramipril in their food $(0.0025 \%$ ramipril by weight) from 6 to 10 weeks of age. Measured dosages of ramipril were: WKY rats $2.94 \pm 0.16 \mathrm{mg} / \mathrm{kg} /$ day; SHRSP, $3.32 \pm 0.18 \mathrm{mg} / \mathrm{kg} /$ day $(\mathrm{P}>0.05)$. All other rats received control food. After treatment from 6 to 10 weeks of age, the ramipril-treated rats were switched to control food for the remainder of the experiment. Blood pressures were measured weekly by an indirect tail cuff method in conscious, restrained rats.

\subsection{Isolated tissue bath protocol}

Rats were killed with a lethal dose of sodium pentobarbital $(60-80 \mathrm{mg} / \mathrm{kg})$ and tail arteries were dissected from the animal. Arteries were cut helically into strips $(0.7 \times 10 \mathrm{~mm})$ and tied with surgical silk at both ends. The strips were mounted in $50 \mathrm{ml}$ organ baths containing physiological salt solution (PSS); one tissue end was tied to a stationary stainless steel rod, the other to an isometric force transducer. PSS was maintained at $37^{\circ} \mathrm{C}$ and aerated with $95 \% \mathrm{O}_{2}-5 \% \mathrm{CO}_{2}$. The composition of PSS was as follows (millimolar): $\mathrm{NaCl}, 130 ; \mathrm{KCl}, 4.7$; $\mathrm{KH}_{2} \mathrm{PO}_{4}, 1.18 ; \mathrm{MgSO}_{4} \cdot 7 \mathrm{H}_{2} \mathrm{O}, 1.17 ; \mathrm{NaHCO}_{3}, 14.9$; $\mathrm{CaCl}_{2} \cdot 2 \mathrm{H}_{2} \mathrm{O}$, 1.60; dextrose, 5.5; and $\mathrm{CaNa}_{2}$ EDTA, 0.03 .

\subsection{Rat tail artery}

Once mounted in the tissue baths, an optimum passive force $(0.6 \mathrm{~g})$ was applied to the tail arteries; the vessels were then allowed to equilibrate $60-90 \mathrm{~min}$ before exposure to compounds. Cocaine $\left(10^{-6} \mathrm{M}\right)$ was included to minimize neuronal uptake of select agonists. Contractile responses to agonists were measured in a non-cumulative fashion. Each concentration of agonist was added to the bath and remained in the bath for $20 \mathrm{~min}$. Agonists were then rinsed from the bath, and the arteries were allowed to return to baseline tension before addition of the next agonist concentration. In some studies, antagonists were added $1 \mathrm{~h}$ before agonist addition. Force generation was measured as the initial response. Oscillatory activity was defined as the number of times tension crossed over a line of zero, which bisected the oscillations, in the last 
$10 \mathrm{~min}$ of a $20 \mathrm{~min}$ incubation with the agonist (see fig. $1 \mathrm{~B}$ for further description).

In experiments dealing with antihypertensive treatment, it was determined whether clonidine $\left(10^{-5} \mathrm{M}\right)$ induced oscillations in tail artery from control, hydralazine/hydrochlorothiazide- or ramipril-treated WKY and SHRSP.

\subsection{Analyses and statistics}

Data are reported as a mean \pm standard error of the mean (S.E.M.) for the number of animals indicated in the parentheses $(\mathrm{N}) . \mathrm{EC}_{50}$ values, the concentration of agonist necessary to produce a half-maximal response, were calculated using the following algorithm:

Response $=\frac{\text { Maximal }}{\left(1+\mathrm{EC}_{50} /[\mathrm{A}]\right)}$

where Response $=$ oscillatory response (zero crossings $/ 10 \mathrm{~min})$, Maximal $=$ maximum oscillatory response (maximum zero crossings $/ 10 \mathrm{~min}$ ) and $[\mathrm{A}]=$ agonist concentration. Estimates of $\mathrm{IC}_{50}$ values, the concentration of antagonists necessary to depress the oscillatory response to a half-maximal level, were calculated in a similar fashion except Response = percent inhibition of norepinephrine $\left(3 \times 10^{-7} \mathrm{M}\right)$-induced oscillatory response, Maximum $=$ maximum percent inhibition of norepinephrine-induced oscillation and $[\mathrm{A}]=$ antagonist concentration. An $\mathrm{IC}_{50}$ value for prazosin could not be calculated as prazosin did not completely abolish the norepinephrine-induced oscillatory contractions up to $3 \times 10^{-6} \mathrm{M}$. Rat brain radioligand binding affinity values used in the correlations were taken from the literature (Rouot et al., 1982).

When appropriate, Student's t-tests, linear regression, weighted logistical modelling with diagnostic methods, analysis of variance and Tukey's post hoc test were used. A P value less than 0.05 was considered statistically significant.

\subsection{Drugs}

The following drugs were used: clonidine hydrochloride (Boehringer Ingelheim, Rigefield, CT, USA), epinephrine bitartrate monohydrate, hydralazine, hydrochlorothiazide (Sigma, Chemical Co., St. Louis, USA), guanabenz acetate (Wyeth, Philadephlphia, PN, USA), methoxamine hydrochloride (Burroughs Wellcome Co., Research Triangle Park, NC, USA), (1)norepinephrine hydrochloride (Calbiochem, San Diegeo, CA, USA), phenylephrine hydrochloride (Sigma, St. Louis, MO, USA), yohimbine hydrochloride (Amend Drug and Chemical Co., New York, NY, USA) and ramipril (provided by Upjohn Co. and Hoechst, Kalamazoo, MI, USA).

\section{Results}

\subsection{NE-induced contractile oscillations in rat tail artery}

Fig. 1A demonstrates the concentration response curves for norepinephrine-induced initial contraction (see inset) in tail artery from WKY and SHRSP. Norepinephrine was slightly but significantly more potent in contracting tail arteries from SHRSP $\left(-\log \mathrm{EC}_{50}=\right.$ $7.85 \pm 0.13)$ than from WKY rats $\left(-\log \mathrm{EC}_{50}=7.48 \pm\right.$ 0.09 ). Inset in fig. $1 \mathrm{~A}$ and $1 \mathrm{~B}$ are tracings of norepinephrine $\left(5.9 \times 10^{-8} \mathrm{M}\right)$-induced contraction in tail arteries from WKY and SHRSP. Note that at similar magnitudes of contraction, tail artery from SHRSP displays marked oscillatory contractions while the contraction induced by norepinephrine in tail artery from WKY rats is steadily maintained. The method used to analyze the oscillatory response is also described in fig. 1B. A line that bisects the contractile oscillations was drawn (fig. 1B, inset) and termed the zero-line. The oscillatory response of the tail artery was then quantified as the number of times the tension passed through the zero-line in the last $10 \mathrm{~min}$ of a $20 \mathrm{~min}$ incubation period with norepinephrine. The resultant concentra-

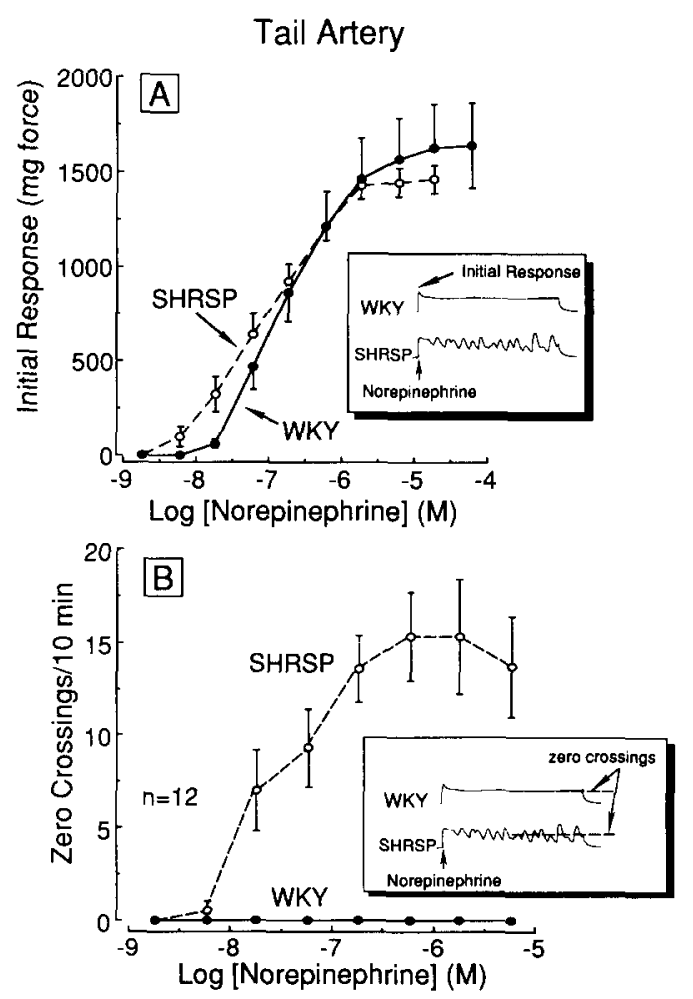

Fig. 1. (A) Concentration-response curves for norepinephrine-induced initial contractions in tail arteries from WKY and SHRSP rats. Inset: tracings demonstrating position of initial contractile response. (B) Concentration-response curves for norepinephrine-induced oscillatory contractions in tail arteries from WKY and SHRSP rats. Points represent means \pm S.E.M for the number of tissues indicated in parentheses. Inset: Tracings demonstrating position of the zero-line which was used to quantitate oscillatory contractions. 


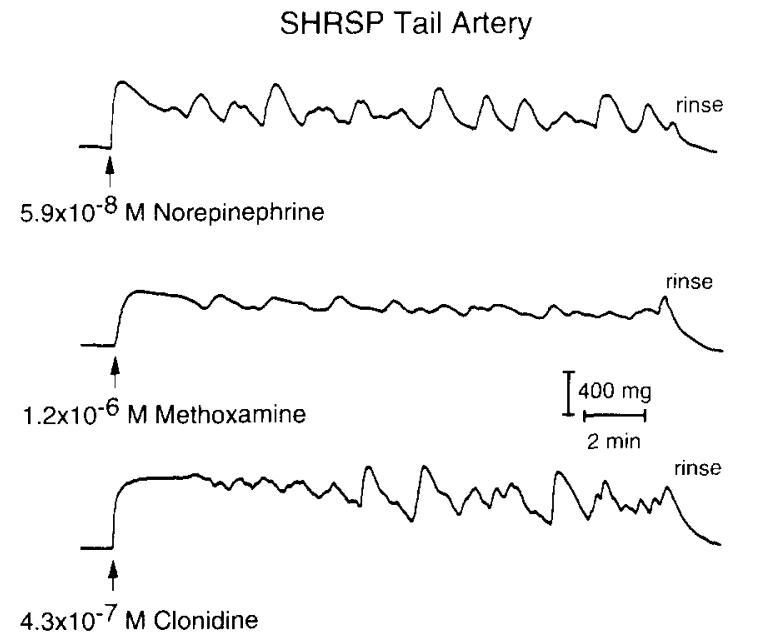

Fig. 2. Representative tracings of the contractile responses of tail artery from SHRSP to the non-selective $\alpha$-adrenoceptor agonist norepinephrine, the $\alpha_{1}$-adrenoceptor agonist methoxamine and the $\alpha_{2}$-adrenoceptor agonist clonidine. Contractions are displayed as change in force $(\mathrm{g}$ ) over time ( $\mathrm{min})$.

tion response curve for norepinephrine-induced oscillations in the tail artery is shown in fig. 1B. Only arteries from SHRSP responded with oscillations to norepinephrine in a concentration-dependent manner.

\subsection{Adrenergic pharmacology of receptor mediating norepinephrine-induced oscillations in tail arteries}

Agonists other than NE also induced an oscillatory response in the tail artery. In concentrations producing a similar magnitude of contraction to norepinephrine $\left(5.9 \times 10^{-8} \mathrm{M}\right)$, both the $\alpha_{1}$-adrenoceptor agonist methoxamine $\left(1.2 \times 10^{-6} \mathrm{M}\right)$ and the $\alpha_{2}$-adrenoceptor agonist clonidine $\left(4.3 \times 10^{-7} \mathrm{M}\right)$ caused contractile oscillations (fig. 2). Fig. 3 compares the concentrationdependent oscillatory contractions induced by various adrenoceptor agonists. Note that agonists possessing

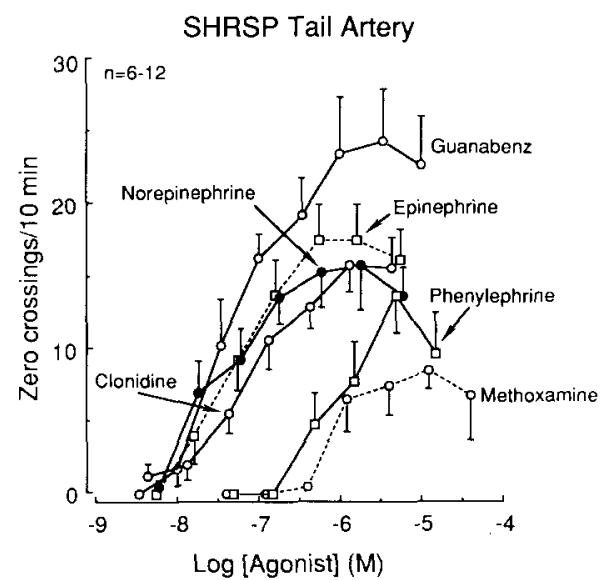

Fig. 3. Concentration-response curves for various adrenoceptor agonists in inducing contractile oscillations in tail arteries from SHRSP.

Points represent means \pm S.E.M. for number of tissues indicated.
SHRSP Tail Artery
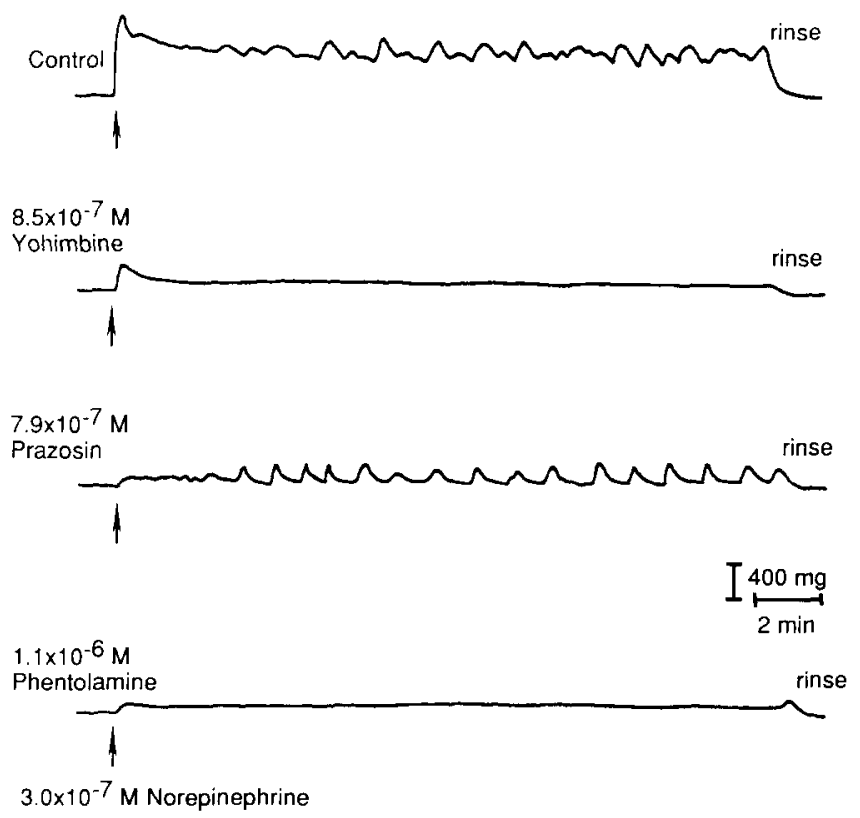

Fig. 4. Contractile responses of tail artery from SHRSP to NE $\left(3.0 \times 10^{-7} \mathrm{M}\right)$ in the absence and presence of the $\alpha_{2}$-adrenoceptor antagonist yohimbine, $\alpha_{1}$-adrenoceptor antagonist prazosin and non-selective $\alpha$-adrenoceptor antagonist phentolamine. Contractions are displayed as change in force $(\mathrm{g})$ over time $(\mathrm{min})$.

significant $\alpha_{2}$-adrenoceptor affinity such as clonidine $\left(-\log \mathrm{EC}_{50}=6.83 \pm 0.13\right)$ and guanabenz $\left(-\log \mathrm{EC}_{50}\right.$ $=7.03 \pm 0.13$ ) were more potent in inducing tail artery oscillations than compounds with significant $\alpha_{1}$-adrenoceptor affinity such as phenylephrine $\left(-\log \mathrm{EC}_{50}=\right.$ $5.57 \pm 0.19)$ and methoxamine $\left(-\log \mathrm{EC}_{50}=5.55 \pm\right.$ 0.09 ). In addition, the compounds with $\alpha_{2}$-adrenoceptor agonist affinity had greater efficacy in causing contractile oscillations in the tail artery than $\alpha_{1}$-adrenoceptor agonists. These data support that $\alpha_{2}$-adrenoceptor stimulation is important in the induction of agonist-induced oscillations.

Moreover, the oscillations induced by a concentration of norepinephrine $\left(3 \times 10^{-7} \mathrm{M}\right)$ which causes maximal oscillatory activity (fig. 1B) were completely blocked by the $\alpha_{2}$-adrenoceptor antagonist yohimbine $\left(8.5 \times 10^{-7} \mathrm{M}\right)$ but not by the $\alpha_{1}$-adrenoceptor antagonist prazosin $\left(7.9 \times 10^{-7} \mathrm{M}\right)$ (fig. 4). The non-selective $\alpha$-adrenoceptor antagonist phentolamine abolished both the maintained tonic and oscillatory contraction induced by norepinephrine. Fig. 5 demonstrates the concentration-dependent inhibition of norepinephrine $\left(3 \times 10^{-7} \mathrm{M}\right)$-induced oscillatory contractions in tail artery from SHRSP by yohimbine, prazosin and phentolamine. Phentolamine $\left(-\log \mathrm{IC}_{50}=6.93 \pm 0.30\right)$ and prazosin demonstrated lesser affinity for the receptor mediating norepinephrine-induced oscillations than the $\alpha_{2}$-adrenoceptor antagonist yohimbine $\left(-\log \mathrm{IC}_{50}=\right.$ $7.85 \pm 0.27$ ); prazosin also incompletely blocked nor- 
epinephrine-induced contractile oscillations in tail artery from SHRSP. These data, together with the agonist data, support that $\alpha_{2}$-adrenoceptors are primarily involved in norepinephrine-induced oscillations in the rat tail artery. When measures of agonist potency $\left(\mathrm{EC}_{50}\right)$ and antagonist affinity $\left(\mathrm{IC}_{50}\right)$ were tested for correlations with $\alpha$-adrenoceptor radioligand binding affinity values from the literature (Rouot et al., 1982), a comparatively strong correlation between compound activity $\left(\mathrm{EC}_{50}, \mathrm{IC}_{50}\right)$ in tail artery and $\alpha_{2}$-adrenoceptor compound binding affinities was revealed (fig. 6). Collectively, these pharmacological data support the involvement of the $\alpha_{2}$-adrenoceptor in norepinephrineinduced oscillations of the rat tail artery. Furthermore, these data support the use of an $\alpha_{2}$-adrenoceptor agonist such as clonidine to investigate the phenomenon of tail artery oscillations.

\subsection{Relationship of blood pressure and oscillations in vascular smooth muscle}

To ascertain whether the phenomenon of agonist-induced oscillations in the rat tail artery was an event dependent on the blood pressure level or angiotensin II production early in life, tail arteries were dissected from WKY and SHRSP which had been treated early in life (6-10 weeks old) with the angiotensin converting enzyme inhibitor ramipril $(3.0 \mathrm{mg} / \mathrm{kg}$ day $)$ or hydralazine/hydrochlorothiazide $(4.0 \mathrm{mg} /$ day $)$. Table $1 \mathrm{docu}-$ ments the mean systolic blood pressures of the rats prior to treatment ( 6 weeks), at the end of treatment (10 weeks) and just prior to experimentation (17 weeks). Note that treatments with both hydrochlorothiazide/ hydralazine and ramipril significantly decreased the blood pressures of SHRSP and WKY rats during the 4 week treatment periods. After treatments were discon-

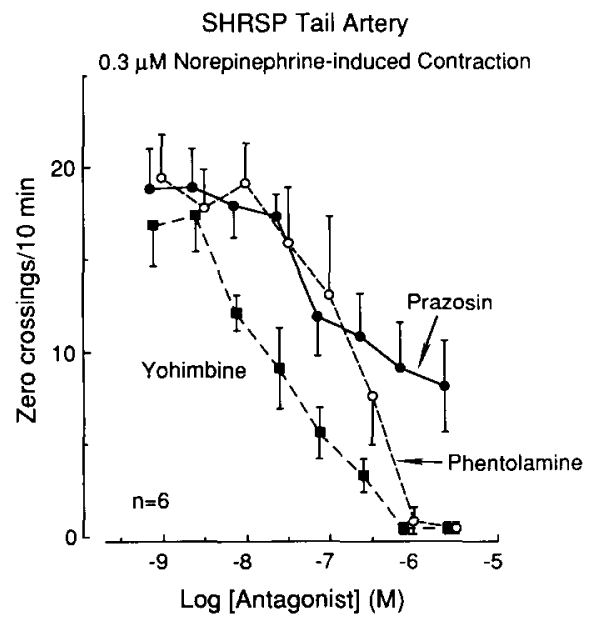

Fig. 5. Concentration-dependent inhibition of NE $\left(3.0 \times 10^{-7} \mathrm{M}\right)$-induced contractile oscillations in tail artery from SHRSP by yohimbine, phentolamine and prazosin. Points represent means \pm S.E.M. for number of tissue indicated.
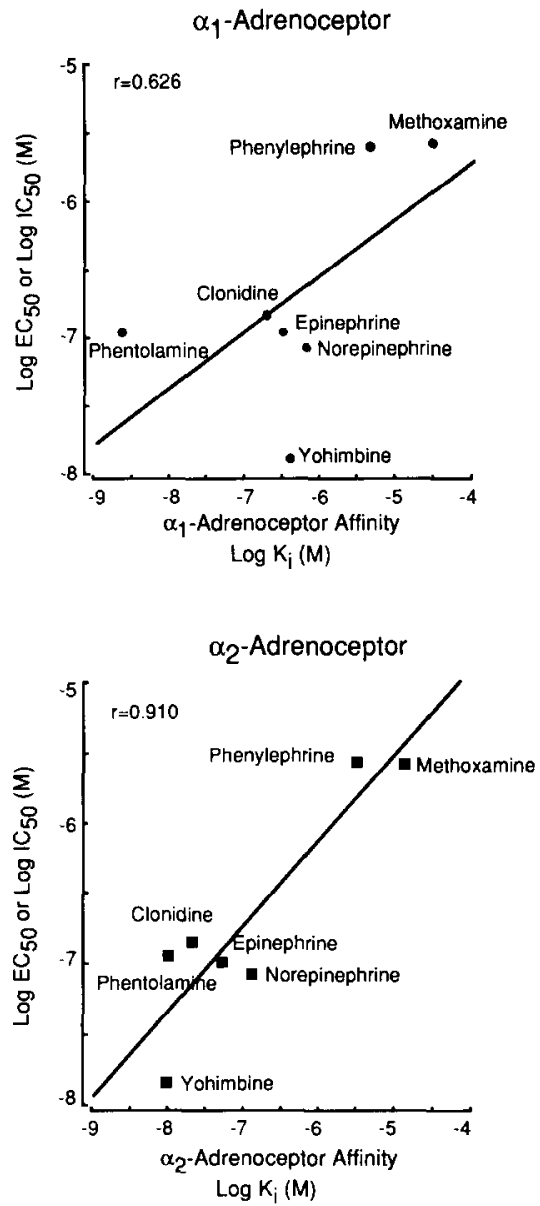

Fig. 6. Correlations between compound activity $\left(\mathrm{EC}_{50}\right.$ or $\left.\mathrm{IC}_{50}\right)$ in tail artery from SHR-SP and reported radioligand binding affinity for the $\alpha_{1}$-adrenoceptor ( $\left[{ }^{3} \mathrm{H}\right]$ prazosin; top) and $\alpha_{2}$-adrenoceptor $\left(\left[{ }^{3} \mathrm{H}\right]\right.$ yohimbine; bottom). Radioligand binding affinities values were taken from Rouot et al. (1982).

tinued, the blood pressure of hydrochlorothiazide/ hydralazine-treated WKY and SHRSP rats and ramipril-treated WKY rats returned to levels equivalent to their respective controls. In contrast, blood pressures of the ramipril-treated SHRSP were significantly depressed compared to SHRSP controls but significantly higher than blood pressures in WKY rats.

Table 1 also demonstrates the percentage of rat tail arteries from the six treatment groups that responded to the $\alpha_{2}$-adrenoceptor agonist clonidine $\left(10^{-5} \mathrm{M}\right)$ with oscillations. If agonist-induced oscillatory contractions of the rat tail artery to agonists were dependent on blood pressure level or angiotensin II production early in life, we would expect the arteries from the ramipril-treated rats to display a lower incidence of oscillatory activity. Virtually none of the tail arteries from WKY rats displayed clonidine-induced oscillatory contractions. In contrast, nearly all tail arteries from SHRSP - regardless of blood pressure - responded to clonidine with oscillations (table 1). Use of logistical modelling determined that the variable blood pressure 


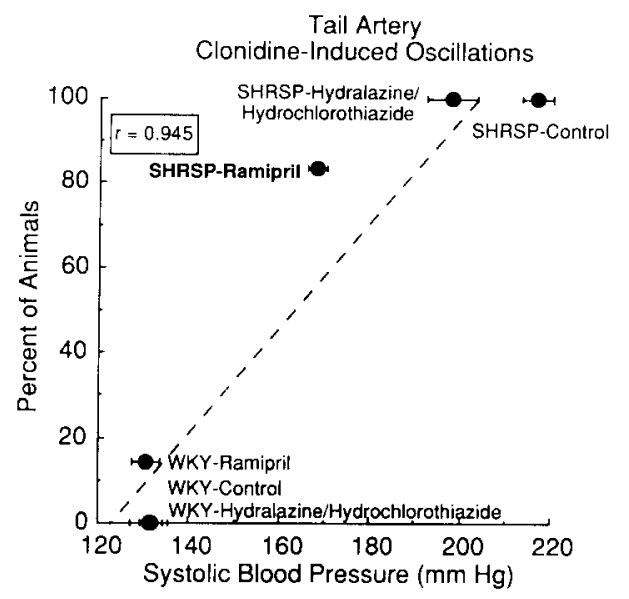

Fig. 7. Correlation between terminal systolic blood pressure $(\mathrm{mm} \mathrm{Hg})$ and percentage of animals whose tail artery oscillated to clonidine $\left(10^{-5}\right.$ M). Points represent means \pm S.E.M. for systolic blood pressure.

was not a suitable predictor of clonidine-induced tail artery oscillations. Furthermore, weighted linear regression between terminal blood pressure and the percentage of animals whose tail arteries oscillated to clonidine (fig. 7) revealed that the SHRSP-ramipril point significantly influenced the slope of the correlation line; this point does not belong on the correlation line. The continued appearance of oscillations in tail artery from ramipril-treated rats would suggest that the decrement ramipril lowered blood pressure (approximately $50 \mathrm{~mm} \mathrm{Hg}$ ) is not influenced by tail artery oscillations, nor does this $50 \mathrm{~mm} \mathrm{Hg}$ drop in pressure alter the appearance of oscillations in tail artery. However, the possibility remains that the remainder of elevated blood pressure $(30-35 \mathrm{mmHg}$ ) in SHRSP may somehow influence tail artery oscillations and vice versa. Nonetheless, these findings suggest that the induction of contractile oscillations in the tail artery of
SHRSP is independent of blood pressure and may be a primary dysfunction in SHRSP. Furthermore, expression of the oscillatory contractions in tail artery of SHRSP does not apparently depend on early angiotensin II production as ramipril did not significantly affect the appearance of oscillatory contractions in the tail artery of SHRSP.

\section{Discussion}

A debate in the study of hypertension exists as to whether the observed changes in reactivity and morphology in vessels from hypertensive subjects are a cause or a result of increased blood pressure. The present studies were performed to investigate further the relationship of blood pressure level and contractile oscillations, vascular events seen in tissues from SHRSP.

In 1985, it was first demonstrated that norepinephrine induced oscillatory contractions in tail arteries from SHRSP but not WKY rats (Myers et al., 1985; Lamb et al., 1985). Since this initial report, norepinephrine-induced tail artery oscillations have been characterized to some extent. First, norepinephrineinduced tail artery oscillations have been observed only in the SHRSP model of hypertension; tail arteries from spontaneously hypertensive rat (SHR), deoxycorticosterone acetate-salt (DOCA-salt) and renal hypertensive rats do not exhibit contractile oscillations to agonists. Tail artery oscillations are also independent of neuronal innervation as acute denervation of tail artery with 6-hydroxydopamine does not alter the ability of norepinephrine to induce oscillations (Myers et al., 1985). Secondly, the cellular mechanisms involved in norepinephrine-induced oscillations in the tail artery have been investigated. Norepinephrine-induced oscil-

TABLE 1

Systolic blood pressures of WKY and SHRSP rats at 6,10 and 17 weeks of age a.

Blood pressures were taken by the tail cuff method in conscious, unanesthetized rats. Values are presented as means \pm S.E.M. for the number of animals indicated in parentheses.

\begin{tabular}{|c|c|c|c|c|}
\hline GROUP & $\begin{array}{l}6 \text { weeks } \\
\text { (prior to treatment) }\end{array}$ & $\begin{array}{l}10 \text { weeks } \\
\text { (end of treatment) }\end{array}$ & $\begin{array}{l}17 \text { weeks } \\
\text { (prior to experimentation) }\end{array}$ & $\begin{array}{l}\text { Number of tail arteries } \\
\text { displaying oscillations }\end{array}$ \\
\hline WKY-control (7) & $101 \pm 8$ & $136 \pm 5$ & $131 \pm 4$ & $0 / 7$ \\
\hline $\begin{array}{l}\text { WKY-hydralazine / } \\
\text { hydrochlorothiazide (6) }\end{array}$ & $96 \pm 7$ & $105 \pm 5^{b}$ & $132 \pm 2$ & $0 / 5^{d}$ \\
\hline WKY-ramipril (8) & $103 \pm 6$ & $109 \pm 4^{b}$ & $131 \pm 3$ & $1 / 7^{d}$ \\
\hline $\begin{array}{l}\text { SHRSP-control (8) } \\
\text { SHRSP-hydralazine }\end{array}$ & $119 \pm 6$ & $174 \pm 4^{c}$ & $217 \pm 4^{c}$ & $8 / 8$ \\
\hline $\begin{array}{l}\text { SHRSP-hydralazine / } \\
\text { hydrochlorothiazide (5) } \\
\text { SHRSP-ramipril (8) }\end{array}$ & $\begin{array}{l}116 \pm 4 \\
124+4\end{array}$ & $\begin{array}{l}113 \pm 9^{b} \\
129+3^{b, c}\end{array}$ & $198 \pm 6^{c}$ & $3 / 3^{d}$ \\
\hline SHRSP-ramipril (8) & $124 \pm 4$ & $129 \pm 3^{n, c}$ & $168 \pm 2^{b, c}$ & $5 / 6^{d}$ \\
\hline
\end{tabular}

${ }^{a}$ A portion of these data are included for descriptive purposes only and are fully discussed in a study submitted by Traub et al. (submitted March 1993); ${ }^{b}$ values are statistically different from respective controls; ${ }^{c}$ values are statistically greater than respective WKY controls; ${ }^{d}$ missing values due to sampling error. 
lations in tail artery from SHRSP depend on access to extracellular $\mathrm{Ca}^{2+}$. This is supported by the fact that (1) norepinephrine-induced oscillations do not occur when extracellular $\mathrm{Ca}^{2+}$ is removed from surrounding buffer (Lamb et al., 1985); and (2) the $\mathrm{Ca}^{2+}$ blockers D-600 (Myers et al., 1985), nifedipine (Lamb et al., 1985) and felodipine (Bruner and Webb, 1989) block norepinephrine-induced oscillations in the tail artery. Similar experiments demonstrated that the non-selective $\mathrm{K}^{+}$channel antagonists quinidine, sparteine and barium also antagonized norepinephrine-induced oscillations (Lamb and Webb, 1989a). In other studies, it was demonstrated that the $\mathrm{Ca}^{2+}$-dependent $\mathrm{K}^{+}$channel blockers apamin and charybdotoxin did not alter norepinephrine-induced oscillations (Lamb and Webb, 1989a). Collectively, these data suggest that norepinephrine-induced oscillations depend upon the influx of $\mathrm{Ca}^{2+}$ and changes in $\mathrm{K}^{+}$movement. Nonetheless, the oscillations are apparently not initiated by the activation of $\mathrm{Ca}^{2+}$-dependent $\mathrm{K}^{+}$channels as neither apamin nor charybdotoxin affected the contractile response. It is not yet clear how these ion movements integrate to result in norepinephrine-induced oscillations. However, using simultaneous measurement of isometric force generation and membrane potential, Lamb and Webb (1989b) have demonstrated that the norepinephrine-induced oscillations in tail arteries from SHRSP were associated with regenerating depolarizations/repolarizations. Thus, the norepinephrine-induced oscillations in tail arteries from SHRSP are rhythmical contractions associated with regenerative, electrical events.

In the present study, we have explored the pharmacology of the adrenoceptor which mediates norepinephrine-induced oscillations of tail arteries from SHRSP. The finding that the $\alpha_{2}$-adrenoceptor agonists clonidine and guanabenz were potent in stimulating oscillations and that the $\alpha_{2}$-adrenoceptor antagonist yohimbine blocked the contractile receptor with high affinity supports that the adrenoceptor mediating contractile oscillations in tail arteries is in the $\alpha_{2}$-adrenoceptor family. This is further supported by the lesser effects of the $\alpha_{1}$-adrenoceptor agonists methoxamine and phenylephrine as well as the relatively lower affinity that prazosin demonstrated for the contractile receptor.

The presence of extrajunctional $\alpha_{2}$-adrenoceptors has been demonstrated in vascular smooth muscle such as the dog saphenous vein (Langer et al., 1980). In the rat tail artery, there appears to be a mix of both $\alpha_{2}-$ and $\alpha_{1}$-adrenoceptors (Langer and Shepperson, 1982; Medgett and Langer, 1984; Rajanayagam and Medgett, 1987; Xiao and Rand, 1989a,b). In the perfused rat tail artery, Sulpizio and Hieble have demonstrated that the $\alpha$-adrenoceptor mediating electrical field-stimulated contraction of the tail artery from normotensive rats is of the $\alpha_{1}$-adrenoceptor family (Sulpizio and Hieble, 1991). Thus, those receptors immediately exposed to norepinephrine released from nerves appear to be $\alpha_{1}$ adrenoceptors. The rat tail artery contraction to exogenously administered norepinephrine could be blocked by both $\alpha_{1}$ - and $\alpha_{2}$-adrenoceptor antagonists (Langer and Shepperson, 1982; Medgett and Langer, 1984). Thus, the mixed response of $\alpha_{1}$ - and $\alpha_{2}$-adrenoceptors in the rat tail artery appears to be similar to the situation in the dog saphenous vein (Langer et al., 1980). Weiss et al. (1984) demonstrated an increased population of $\alpha_{2}$-adrenoceptors in both the brain (amygdala) and tail artery of the SHR when compared to tissues from WKY rats. As SHRSP are a substrain of the SHR, we can postulate that an increased $\alpha_{2}$ adrenoceptor density may also be found in SHRSP. Thus, one possible reason for the observed lack of oscillations in tail artery from WKY may be an $\alpha_{2}$ adrenoceptor population too small for an oscillation to be acheived. This postulate is supported by studies from Medgett et al. (1984) in which tail artery from SHR possessed a greater $\alpha_{2}$-adrenoceptor population than tail artery from WKY rats. While $\alpha_{2}$-adrenoceptor subtypes exist (Bylund, 1992), we have not investigated which $\alpha_{2}$-adrenoceptor(s) were in the tail artery. Nevertheless, the present studies demonstrate that the primary adrenoceptor involved in norepinephrine-induced oscillatory responses is most likely an $\alpha_{2}$-adrenoceptor.

We next investigated the relationship of this oscillatory event in the tail artery with blood pressure level. This was done for two reasons. First, it had previously been demonstrated that norepinephrine-induced oscillations in SHRSP are genetically associated with elevated blood pressure in the SHRSP. Specifically, a study of the relationship between blood pressure and tail artery oscillation in genetic crosses $\left(F_{1}\right)$ of SHRSP and WKY rats indicated that the gene(s) which result in expression of oscillatory activity (a phenotype) may also be a determinant of high blood pressure (Bruner et al., 1986). Furthermore, when the hypertension of SHRSP was chronically treated with the vasodilator hydralazine and diuretic hydrochlorothiazide, blood pressure levels were lowered in SHRSP but the oscillatory contraction of the tail artery remained (Bruner and Webb, 1987). Collectively, these studies were the first to suggest that norepinephrine-induced oscillatory contraction in tail artery of SHRSP may not be an effect secondary to development of high blood pressure, but an intrinsic change of the vessel. Our second reason for the present studies was to investigate a hypothesis proposed by Harrap et al. (1990). Harrap et al. (1990) demonstrated that treatment of young, genetically hypertensive rats with the angiotensin converting enzyme inhibitor perindopril at a young age (2-10 weeks) resulted in a significant and apparently perma- 
nent decrease in blood pressure. These results were interpreted to mean that a 'critical period' for highblood pressure development exists early in life. A 'gene-switching' hypothesis was proposed; early in life, the development of high blood pressure may be dependent on the production and manifestation of the effects of angiotensin II. Angiotensin II may 'switch' on and/or off particular genes, thereby initiating a series of events that ultimately leads to the expression of hypertension. By inhibiting the production of angiotensin II with perindopril, these genes may never get 'switched'; thus, hypertension may not be expressed. We have reproduced these studies using the angiotensin converting enzyme inhibitor ramipril.

During the 4 week treatment period, both ramipril and hydralazine/hydrochlorothiazide treatment significantly lowered blood pressures from control SHRSP levels (table 1). Upon discontinuation of treatment, blood pressures of the hydralazine/hydrochlorothiazide group rebounded to levels similar to those of the control SHRSP. In contrast, blood pressures of the ramipril-treated SHRSP group remained significantly reduced when compared to control SHRSP. Thus, ramipril, like perindopril, appears to have long-term affects on the expression of high blood pressure. The hypothesis we then tested was whether clonidine-induced oscillations in the tail artery would still occur in these putatively 'switched' animals. If agonist-induced oscillations in the tail artery were under control of angiotensin II expression, we would expect that angiotensin converting enzyme inhibition would turn off the adrenoceptor-mediated oscillation. Clonidine $\left(10^{-5}\right.$ M)-induced oscillations in a statistically similar percentage of rat tail arteries isolated from ramipriltreated SHRSP and untreated SHRSP; thus, the appearance of agonist-induced oscillations in the tail artery from SHRSP is most likely an event independent of angiotensin II generation during the proposed critical period. Furthermore, the logistic model used to examine the relationship of clonidine-induced oscillation of rat tail artery and blood pressure concluded that blood pressure was not the best indicator of whether a tail artery would oscillate to clonidine. These results support previous indications (Bruner et al., 1986; Bruner and Webb, 1987) that the SHRSP tail artery oscillation stimulated by norepinephrine may truly be a primary dysfunction of the SHRSP and not a result of elevated blood pressure.

The studies in the present report indirectly support the association of oscillatory contractions and elevated blood pressure, and the hypothesis that oscillatory contractions such as those described above may characterize hypertension. For example, Meininger et al. (1984) estimated that in renal hypertensive rats, 30 percent of the increased hindquarter vascular resistance was caused by rhythmic arteriolar vasomotor activity. In the human renal circulation, stimulus-induced flow responses were oscillatory (Hollenberg and Sandor, 1984; Hollenberg, 1987). Comparable to the agonist-induced oscillations in the tail artery from SHRSP (Myers et al., 1985; Lamb et al., 1985), oscillatory flow responses in human renal circulation can be inhibited by $\mathrm{Ca}^{2+}$ channel antagonists. Recently, Webb et al. (1992) demonstrated that agonists - serotonin and endothelin - induced oscillatory contractions in a greater percentage of vertebral arteries from hypertensive than in normotensive individuals. Again, these oscillations were similar to those observed in tail artery from SHRSP in that the oscillations were dependent on extracellular $\mathrm{Ca}^{2+}$ and blocked by the L-type voltage sensitive $\mathrm{Ca}^{2+}$ channel antagonists verapamil and nifedipine.

In summary, we have performed a series of experiments using the genetically hypertensive SHRSP and normotensive WKY rats that investigates: (1) the complex relationship of altered vascular reactivity with blood pressure level; and (2) a recently proposed hypothesis concerning gene 'switching' in genetic hypertension. First we determined that the primary adrenoceptor mediating the unique phenomenon of norepinephrine-induced oscillations in tail arteries from SHRSP is most likely an $\alpha_{2}$-adrenoceptor. Second, we found that although the angiotensin converting enzyme inhibitor ramipril lowered blood pressures of SHRSP significantly, it did not alter the appearance of clonidine-induced tail artery oscillations in SHRSP, thereby supporting the independence of this vascular event from blood pressure and early angiotensin II production.

\section{Acknowledgements}

SWW was supported by a Research Fellowship from the American Heart Association of Michigan. This work was supported by The National Institutes of Health HL-18575. The authors would also like to thank Dr. Anthony Schork for help with statistical analyses.

\section{References}

Bohr, D.F. and R.C. Webb, 1988, Vascular 'smooth muscle membrane in hypertension, Ann. Rev. Pharmacol. Toxicol. 28, 389.

Bruner, C.A., J.H. Myers, C.G. Sing, P.T. Jokelainen and R.C. Webb, 1986, Genetic association of hypertension and vascular changes in stroke-prone spontaneously hypertensive rats, Hypertension 8,904

Bruner, C.A. and R.C. Webb, 1987, Effect of antihypertensive therapy on a vascular change in genetically hypertensive rats, Clin. Exp. Theory Practice A9 (11), 1745

Bruner, C.A. and R.C. Webb, 1989, Effect of felodipine on blood pressure and vascular reactivity in stroke-prone spontaneously hypertensive rats, J. Hypertens. 7, 31.

Bylund, D.B., 1992, Subtypes of $\alpha_{1}$ - and $\alpha_{2}$-adrenergic receptors, FASEB J. 6, 832 . 
Gabbianai, G., G. Elemer, C. Guelpa, M.B. Valloton, M.-C. Badonnel and I. Huttner, 1979, Morphologic and functional changes of the aortic intima during experimental hypertension, Am. J, Pathol. $96,399$.

Grunwald, J., H. Robenek, J. Mey and W.H. Hauss, 1982, In vivo and in vitro cellular changes in experimental hypertension: electronmicroscopic and morphometric studies of aortic smooth muscle cells, Exp. Mol. Pathol. 36, 164.

Guyton, J.R., D.T. Dao, K.L. Lindsay and A.A. Taylor, 1990, Ultrastructure of hypertensive rat aorta: increased basement membrane-like material, Hypertension 15, 56.

Harrap, S.B., W.M. Van der Merwe, S.A. Griffin, F. Macpherson and L.F. Lever, 1990, Brief angiotensin converting enzyme inhibitor treatment in young spontaneously hypertensive rats reduces blood pressure long-term, Hypertension 16, 603 .

Hollenberg, N.K., 1987, Vasodilators, antihypertensive therapy and the kidney, Am. J. Cardiol. 60, 571

Hollenberg, N.K. and T. Sandor, 1984, Vasomotion of renal blood flow in essential hypertension: oscillations in xenon transit, Hypertension, 6, 579.

Lamb, F.S. and R.C. Webb, 1989a, Potassium conductance and oscillatory contractions in tail arteries from genetically hypertensive rats, J. Hypertens. 7, 457.

Lamb, F.S. and R.C. Webb, 1989b, Regenerative electrical activity and arterial contraction in hypertensive rats, Hypertension 13, 70.

Lamb, F.S., J.H. Myers, M.N. Hamlin and R.C. Webb, 1985, Oscillatory contractions in tail arteries from hypertensive rats, Hypertension 7 (Suppl. 1), I-25.

Langer, S.Z. and N.B. Shepperson, 1982, Recent development in vascular smooth muscle pharmacology-The postynaptic $\alpha_{2}$ adrenoceptor, Trends Pharmacol. Sci. 3, 440.

Langer, S.Z., R. Massingham and N.B. Shepperson, 1980, Presence of postsynaptic $\alpha_{2}$-adrenoceptors of predominantly extra-synaptic location in the vascular smooth muscle of the dog hind limb, Clin. Sci. (Oxford) 59, 225s.

Medgett, I.C., P.E. Hicks and S.Z. Langer, 1984, Smooth muscle alpha-2 adrenoceptors mediate vasoconstrictor responses to exogenous norepinephrine and to sympathetic stimulation to a greater extent in spontaneously hypertensive than in Wistar-Kyoto rat tail arteries, J. Pharmacol. Exp. Ther. 231, 159.

Medgett, I.C. and S.Z. Langer, 1984, Heterogeneity of smooth muscle alpha adrenoceptors in rat tail artery in vitro, J. Pharmacol. Exp. Ther. 229, 823.
Meininger, G.A., V.M. Lubrano and H.J. Granger, 1984, Hemodynamic and microvascular responses in the hindquarters during the development of renal hypertension in rats: evidence for the involvement of an autoregulatory component, Circ. Res. 55, 609.

Myers, J.H., F.S. Lamb and R.C. Webb, 1985, Norepinephrine-induced phasic activity in tail arteries from genetically hypertensive rats, Am. J. Physiol. 284, H419.

Owens, G.K. and S.M. Schwartz, 1982, Alterations in vascular smooth muscle mass in the spontaneously hypertensive rat: role of cellular hypertrophy, hyperpoloidy and hyperplasia, Circ. Res. 56, 525.

Rajanayagam, M.A.S. and I.C. Medgett, 1987, Greater activation of smooth muscle alpha-2-adrenoceptors by epinephrine in distal than in proximal segments of rat tail artery, J. Pharmacol. Exp. Ther. 240, 1989.

Rouot, B., M.C. Quennedey and J. Schwartz, 1982, Characteristics of the $\left[{ }^{3} \mathrm{H}\right]$-yohimbine binding on rat brain $\alpha_{2}$-adrenoceptors, Naunyn-Schmied. Arch. Pharmacol. 321, 253.

Stills, W.J.S., 1979, The effect of chronic hypertension on the aortic intima of the rat, Exp. Mol. Pathol. 31, 1.

Sulpizio, A. and J.P. Hieble, 1991, Lack of a pharmacological distinction between alpha-1 adrenoceptors mediating intracellular calcium-dependent and independent contractions to sympathetic nerve stimulation in the perfused rat caudal artery, J. Pharmacol. Exp. Ther. 257, 1045.

Todd, P.A. and P. Benfield, 1990, Ramipril: a review of its pharmacological properties and therapeutic efficacy in cardiovascular disorders, Drugs 39, 1.

Webb, R.C., K.D. Schreur and S.M. Papadopoulos, 1992, Oscillatory contractions in vertebral arteries from hypertensive subjects, Clin. Physiol. 12, 69.

Weiss, R.J., R.C. Webb and C.B. Smith, 1984, Comparison of alpha2 adrenoreceptors on arterial smooth muscle and brain homogenates from Spontaneously Hypertensive and Wistar-Kyoto normotensive rats, J. Hypertens. 2, 249.

Xiao, X.-H. and M.J. Rand, 1989a, $\alpha_{2}$ adrenoceptor agonists enhance responses to certain other vasoconstrictor agonists in the rat tail artery, $\mathrm{Br}$. J. Pharmacol. 96, 539.

Xiao, X.-H. and M.J. Rand, 1989b, $\alpha_{2}$-adrenoceptor agonists enhance vasoconstrictor responses to $\alpha_{1}$-adrenoceptor agonists in the rat tail artery by increasing the influx of $\mathrm{Ca}^{2+}, \mathrm{Br}$. J. Pharmacol. $98,1032$. 